\title{
HLX affects cell cycle and proliferation in AML cells via the JAK/STAT signaling pathway
}

\author{
XIA-YIN ZHU ${ }^{1}$, QUN-YI GUO ${ }^{1}$, MIN ZHU $^{2}$, BAO-GUO CHEN ${ }^{2}$, LING-YAN WANG ${ }^{1}$, \\ DAN-QIONG ZHANG ${ }^{1}$, LI ZHANG ${ }^{1}$, YAN-PING SHAO ${ }^{1}$ and WEN-DA LUO ${ }^{1}$ \\ Departments of ${ }^{1}$ Hematology and ${ }^{2}$ Central Laboratory, Taizhou Hospital of Zhejiang, \\ Wenzhou Medical College, Taizhou, Zhejiang 317000, P.R. China
}

Received January 5, 2020; Accepted May 13, 2020

DOI: $10.3892 / \mathrm{ol} .2020 .11718$

\begin{abstract}
Acute myelogenous leukemia (AML) is a class of malignant tumors derived from hematopoietic stem or progenitor cells. The H2.0-like homeobox gene (HLX) encodes transcription factors that function in promoting normal hematopoietic cell proliferation and tumor immunity. The present study analyzed the effect of downregulating the HLX on cell cycle distribution and cell proliferation in AML. Moreover, the current study detected changes in the expression of genes and proteins in the Janus kinase (JAK)/STAT signaling pathway to investigate the mechanism of the action of HLX in tumor immunity in AML. HLX expression in AML cell lines was silenced using small interfering siRNA, and MTS/PMS-assay colorimetric assays were used to assess the effect of knockdown of HLX on AML cell proliferation. Flow cytometry was used to analyze changes in cell cycle distribution, while reverse transcription-quantitative PCR and western blotting were used to detect changes in the expression levels of key components of the JAK/STAT signaling pathway, such as p21-activated kinase 1 (PAK1), neuropilin 1 (NRP1), B-cell translocation gene 1 (BTG1) and STAT5. It was found that HLX was differentially expressed in AML cell lines of various subtypes, and HLX expression was higher in the AML/M3 subtype NB4 cell line compared with the control group. Knockdown of HLX in NB4 cells significantly inhibited cell proliferation and arrested cells in the $\mathrm{G}_{0} / \mathrm{G}_{1}$ phase. Moreover, STAT5 protein expression, as well as NRP1 and PAK1 expression levels were
\end{abstract}

Correspondence to: Dr Wen-Da Luo, Department of Hematology, Taizhou Hospital of Zhejiang, Wenzhou Medical College, 150 Ximen Street, Linhai, Taizhou, Zhejiang 317000, P.R. China E-mail: luowd@enzemed.com

Abbreviations: AML, acute myelogenous leukemia; HLX, H2.0-like homeobox gene; PAK1, p21-activated kinase 1; NRP1, neuropilin 1; BTG1, B-cell translocation gene 1

Key words: NB4 cells, HLX, Janus kinase/STAT, proliferation inhibition downregulated, while BTG1 expression was upregulated when HLX was knocked out by siRNA. Collectively, the results suggested that downregulation of HLX may cause $G_{0} / G_{1}$ phase arrest and inhibit the proliferation of AML cells by activating the JAK/STAT signaling pathway.

\section{Introduction}

The H2.0-like homeobox gene (HLX) encodes transcription factors that play roles in promoting normal hematopoietic cell proliferation and tumor immunity, and the HLX protein plays a key role in the development of cancer (1). HLX is expressed at high levels in various tumor cells but at low levels in normal cells; however, the complete deletion of HLX results in cell death $(2,3)$. A previous study reported that the expression of HLX decreased during CD $34^{+}$hematopoietic stem or progenitor cell differentiation, suggesting that HLX may play a role in maintaining the differentiation potential of hematopoietic stem or progenitor cells (4).

Acute myelogenous leukemia (AML) is a class of malignant tumors derived from hematopoietic stem or progenitor cells. The main treatment methods for AML include chemotherapy, radiotherapy, hematopoietic stem cell transplantation, cell immunotherapy, targeted therapy and traditional Chinese medicine treatment (5). Currently, it is difficult to improve the complete remission rate and long-term survival rate with existing treatment regimens (6). However, research and examination of immunotarget therapy have revealed that tumor immunotherapy and molecular targeted therapy may be potential therapeutic breakthroughs. As previously demonstrated, the aim of Feiji Recipe (Components of this prescription: Astragalus $30 \mathrm{~g}$, Atractylodes macrocephala $15 \mathrm{~g}$, Poria $20 \mathrm{~g}$, American ginseng $10 \mathrm{~g}$, Trichosanthes $30 \mathrm{~g}$, pinellia $12 \mathrm{~g}$, Fritillaria Zhejiang $20 \mathrm{~g}$, yam $15 \mathrm{~g}$, job's tears $30 \mathrm{~g}$, Hedyotis Baihua $30 \mathrm{~g}$, Chonglou $30 \mathrm{~g}$, zhibaibu $15 \mathrm{~g}$, bayzha $15 \mathrm{~g}$, zaojiaoci $30 \mathrm{~g}$, Chenpi $12 \mathrm{~g}$, liquorice $6 \mathrm{~g}$, each patient's dosage and content of the recipe are not exactly the same) in the treatment of lung cancer was to restore the function of T-cells in the cancer microenvironment by interfering with the indoleamine 2,3-dioxygenase pathway (7). In recent years, fms related receptor tyrosine kinase 3 (FLT3), Nucleophosmin 1 (NPM1), DNA (cytosine-5)-methyltransferase 3A (DNMT3a) and isocitrate dehydrogenase (NADP+) 2 (IDH2) have been 
the top four targeted molecules in AML (7). Drug development with NPM1 is difficult, and thus is not considered a candidate (8). Moreover, the mutation of DNMT3AR882H can inhibit the function of the wild-type protein, and the development of inhibitors worsens the disease (7). Thus, FLT3 and IDH2 have become the main focus of drug development. IDH1 and IDH2 have similar molecular structures, and related drugs are also in development (9). Furthermore, molecular targeted therapy has resulted in positive outcomes in the treatment of hematologic malignancies (10). Previous studies have identified HLX, which is highly expressed in AML cells, as a new target for this malignancy and reported that high HLX expression is associated with poor prognosis in patients with AML, but the specific mechanism of HLX gene function in AML remains unknown $(10,11)$.

Previous studies have shown the effect of inhibiting HLX on suppressing the proliferation of leukemia cells and have revealed the relationship between HLX with p21-activated kinase 1 (PAK1) and B-cell translocation gene 1 (BTG1) (10-14); however, to the best of our knowledge, these studies have not examined the Janus kinase (JAK)/STAT signaling pathway. Thus, the present study analyzed HLX expression in every subtype of AML cells, and then focused on the NB4 cell line (AML/M3 subtype) to elucidate the function and possible mechanism of action of HLX in AML.

\section{Materials and methods}

Media and reagents. The human AML KG1a, NB4 and THP-1 cell lines and the human acute lymphoblastic leukemia Jurkat cell line were obtained from the Center Laboratory of Enze Medical Group (Zhejiang, China). RPMI-1640 medium and FBS were obtained from Cytiva. DEPC water, TRIzol ${ }^{\circledR}$, RNAlater Stabilization Solution and Blockit Alexa Fluor Red Fluorescent Oligo were obtained from Thermo Fisher Scientific, Inc. SYBR-Green was obtained from Roche Diagnostics, and PCR reagents were obtained from Axygen (Corning, Inc.). Lipofectamine ${ }^{\circledR}$ RNAiMAX Reagent (www. lifetechnologies.com), STAT5 antibody (cat. no. QC215910), small interfering siRNA-HLX1 and siRNA-HLX2 were obtained from Thermo Fisher Scientific, Inc. The HLX1 antibody (cat. no. GTX87590) was obtained from GeneTex International Corporation. Lipofectamine ${ }^{\circledR} 2000$ was obtained from Invitrogen (Thermo Fisher Scientific, Inc.), and the Cell Cycle Staining kit was obtained from Hangzhou Multi Sciences (Lianke) Biotech Co., Ltd. The FACSCalibur flow cytometer and PCR machine were obtained from BD Biosciences.

Cell culture. The human leukemia cell lines were cultured in RPMI 1640 medium with $10 \%$ FBS in an incubator at $37^{\circ} \mathrm{C}$ with $5 \% \mathrm{CO}_{2}$ and $95 \%$ humidity.

RNA extraction and reverse transcription-quantitative PCR $(R T-q P C R)$. Total RNA was extracted using the Qiagen RNeasy ${ }^{\circledR}$ Mini kit (Qiagen, Inc.) and a RevertAid RT Reverse Transcription kit (Thermo Fisher Scientific, Inc.) was used to reverse transcribe RNA into cDNA at $65^{\circ} \mathrm{C}$ for $5 \mathrm{~min}$. qPCR was performed using the SYBR Premix Ex Tag kit (Takara Bio, Inc.) at $42^{\circ} \mathrm{C}$ for $60 \mathrm{~min}, 70^{\circ} \mathrm{C}$ for $5 \mathrm{~min}$ and $40^{\circ} \mathrm{C}$ for $10 \mathrm{~min}$, for 40 cycles, and an ABI 7500 Sequencing Detection system (Applied Biosystems; Thermo Fisher Scientific, Inc.) according to the manufacturer's protocols. GAPDH was used as a quantitative control gene, and all reactions were performed in triplicate.

The housekeeping gene GAPDH served as the reference gene, and RT-qPCR was performed using an ABI 7500 Sequencing Detection system. The results were calculated using the $2^{-\Delta \Delta \mathrm{Cq}}$ method (15), and the median $\Delta \mathrm{Cq}$ value of GAPDH was used to calculate expression levels in the control group. Primer sequences of genes HLX, PAK1, neuropilin 1 (NRP1) and BTG1 for RT-qPCR were as follows (www. generay.com): HLX: Forward, 5'-ATCTCACTTCCCTGC TAACCG-3' and reverse, 5'-AGAAGCCTCGTTAATGGG ATCT-3'; PAK1: Forward, 5'-CAGCCCCTCCGATGAGAA ATA-3' and reverse, 5'-CAAAACCGACATGAATTGTGT GT-3'; BTG1: Forward, 5'-AGCGGATTGGACTGAGCA G-3' and reverse, 5'-GGTGCTGTTTTGAGTGCTACC-3'; NRP1: Forward, 5'-ACGTGGAAGTCTTCGATGGAG-3' and reverse, 5'-CACCATGTGTTTCGTAGTCAGA-3'; GAPDH: Forward, 5'-CTGGGCTACACTGAGACC-3' and reverse, 5'-AAGTGGTCGTTGAGGGCAATG-3'.

Western blotting. Proteins were extracted from cells in the logarithmic growth phase using RIPA buffer supplied by Enze Medical Group Laboratory. For blocking, 5\% skimmed milk was used at $4^{\circ} \mathrm{C}$ overnight. Protein was determined using a BCA Protein Assay kit (Sangon Biotech Co. Ltd). In total, $50 \mathrm{kDA}$ protein was loaded per lane onto a $5 \%$ gel. The proteins were then transferred to polyvinylidene difluoride (PVDF) membranes $(0.45 \mu \mathrm{m})$. The PVDF membrane was incubated with the following primary antibodies overnight at $4^{\circ} \mathrm{C}$ : HLX1 (cat. no. GTX87590, 1:1,000) and STAT5 (cat. no. QC215910, 1:2,000), obtained from GeneTex International Corporation. Then, the membranes were incubated with HLX secondary antibody [goat anti-rabbit IgG $(\mathrm{H}+\mathrm{L})$ secondary antibody, cat. no. 31460, 1:1,000, Invitrogen; Thermo Fisher Scientific, Inc.], STAT secondary antibody [goat anti-mouse IgG, $\operatorname{Ig} \mathrm{M}(\mathrm{H}+\mathrm{L})$ secondary antibody, cat. no. A-10677, 1:3,000, Invitrogen; Thermo Fisher Scientific, Inc.] for $1 \mathrm{~h}$ at room temperature. Band intensity was semi-quantitatively analyzed using ImageJ software version 1.8.0 (National Institutes of Health).

Construction of HLX-knockdown cells. HLX-specific sequences were designed according to the GenBank database (Invitrogen; Thermo Fisher Scientific, Inc.), and siRNA-HLX1 and siRNA-HLX2 were generated (Table I). AML cells were transfected with siRNA-HLX1, -HLX2 or non-targeting control siRNA-highGC using Lipofectamine ${ }^{\circledR}$ RNAiMAX (www. lifetechnologies.com). The following solutions were used: i) Liquid A, $25 \mu \mathrm{l}$ Opti-MEM Medium + $0.5 \mu \mathrm{l}$ siRNA; and ii) Liquid B, $25 \mu \mathrm{l}$ Opti-MEM + 1.5 $\mu \mathrm{l}$ Lipofectamine. Then, liquid $\mathrm{B}$ was added to liquid $\mathrm{A}$ for $5 \mathrm{~min}$ at room temperature and mixed to obtain Liquid C. Cells were added at a density of $\left(1-4 \times 10^{4}\right)$ to liquid $\mathrm{C}$ after $20 \mathrm{~min}$ at room temperature, followed by incubation for $1-3$ days at $37^{\circ} \mathrm{C}$. The transfected cells were observed and counted manually under the fluorescence microscope at 200x magnification. The cells were divided into the blank control group (no added reagent), the negative control group (transfected with non-specific high-GC siRNA) 
Table I. Sequences of siRNA-HLX1 and siRNA-HLX2.

\begin{tabular}{lccc}
\hline Gene & Cat. no. & Type & Sequence $\left(5^{\prime} \rightarrow 3^{\prime}\right)$ \\
\hline SiRNA-HLX1 & 10620318-296424 E04 & RNA & CCCUUAAACUCGAACCCAAGAAAUU \\
siRNA-HLX2 & 10620319-296424 E05 & RNA & AAUUUCUUGGGUUCGAGUUUAAGGG \\
& 10620318-296424 E06 & RNA & GCUGAGAGAUCUCACUUCCCUGCUA \\
& $10620319-296611$ A12 & RNA & UAGCAGGGAAGUGAGAUCUCUCAGC \\
\hline
\end{tabular}

Supplier: Thermo Fisher Scientific, Inc. siRNA, small interfering RNA; HLX, H2.0-like homeobox gene.

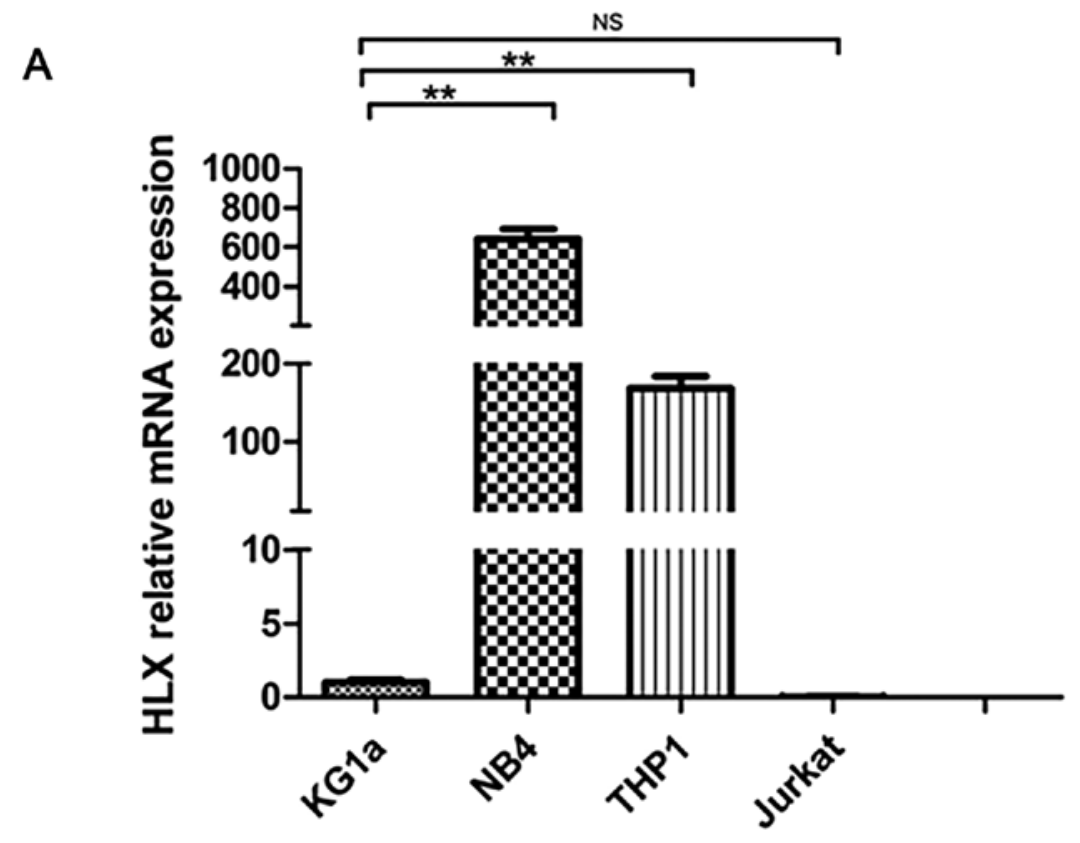

B

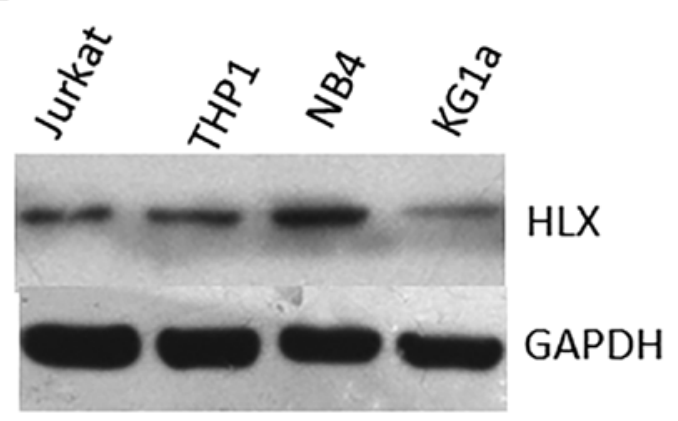

C

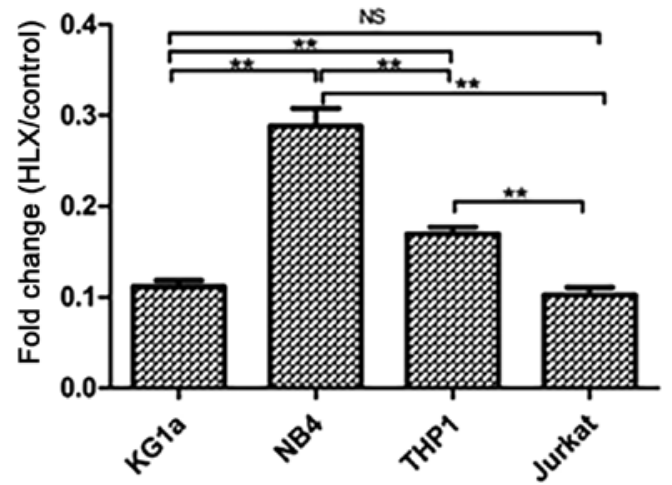

Figure 1. HLX expression in AML cell lines. (A) Comparison of HLX gene expression in different cell lines. (B) Comparison of HLX protein expression in different cell lines. (C) Gray value analysis of western blotting results. ${ }^{* * *} \mathrm{P}<0.01$. NS, not significant; HLX, H2.0-like homeobox gene.

and experimental group (transfected with siRNA-HLX1 or siRNA-HLX2). In total, three wells were used for each group. The transfection efficiency was determined at the time points of $12,24,48$ or $72 \mathrm{~h}$ after transfection, and the experiment was repeated three times.

MTS/PMS assay for cell proliferation. NB4 cells were seeded into 96 -well plates at a density of $1 \times 10^{5}$ cells $/ \mathrm{ml}$. The following groups were analyzed: Negative control group (transfected with non-specific high-GC siRNA) and experimental group (siRNA-HLX1 or siRNA-HLX2).Proliferation was determined using the MTS/PMS Cell Proliferation Assay kit (www. liankebio.com) according to the manufacturer's instructions. The absorbance was measured at $490 \mathrm{~nm}$ on a multi-well plate reader at 12, 24, 48 and $72 \mathrm{~h}$ after transfection. The survival rate was calculated [Survival inhibition rate $=(1$-odvalue 


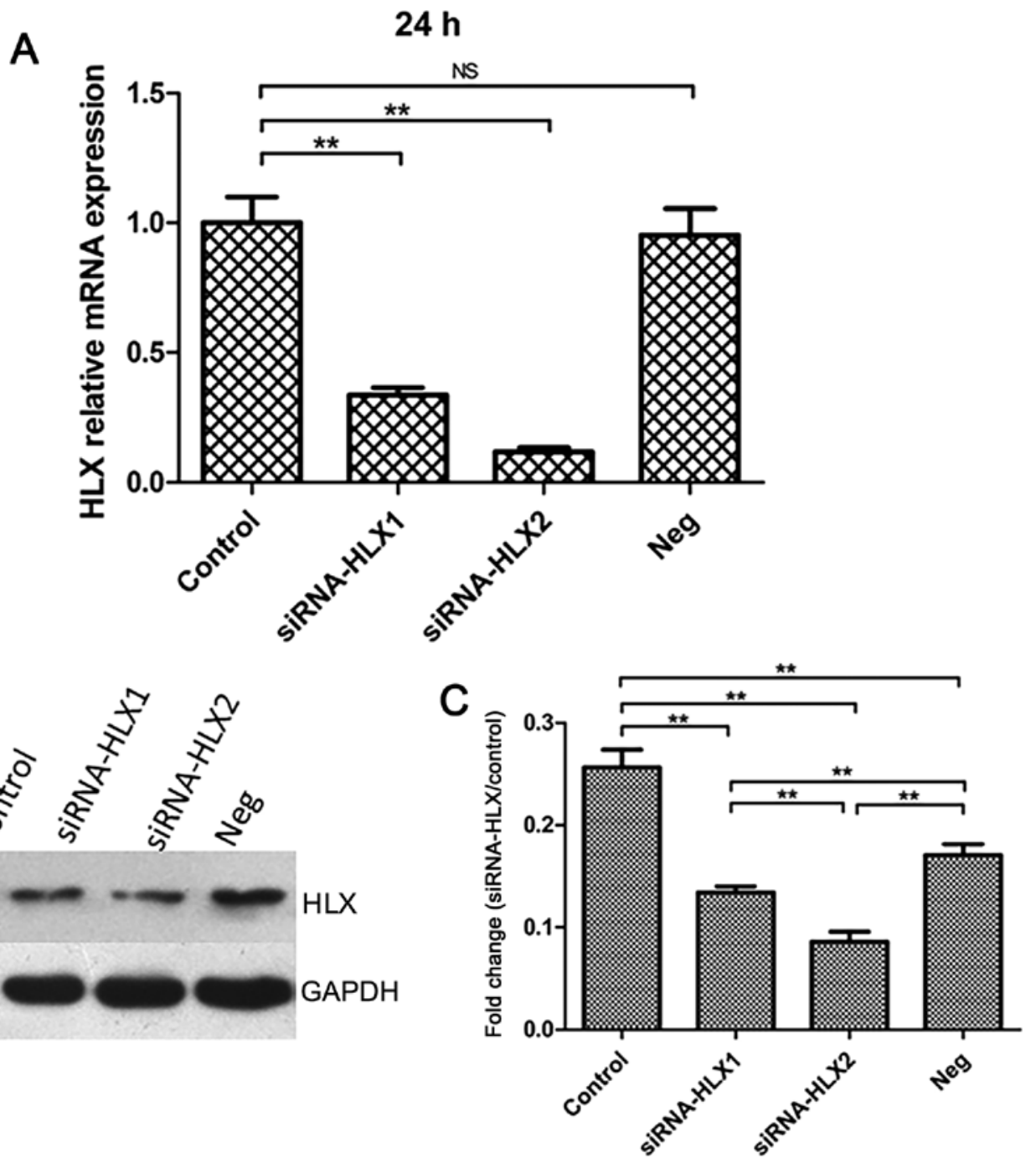

Figure 2. Changes in HLX expression after siRNA transfection of NB4 cells. (A) HLX gene expression was determined by reverse transcription-quantitative $\mathrm{PCR}$ at $24 \mathrm{~h}$ after siRNA transfection. (B) HLX protein expression was detected by western blotting at $24 \mathrm{~h}$ after siRNA transfection. (C) Gray value analysis of western blotting results. ${ }^{* *} \mathrm{P}<0.01$. NS, not significant; HLX, H2.0-like homeobox gene; siRNA, small interfering RNA; Neg, negative control.

of experimental group/odvalue of control group) x100\%], and each assay was performed in triplicate. The results are presented as the mean \pm standard deviation $(\mathrm{SD})$.

Cell cycle assay. NB4 cells were fixed with $70 \%$ ethanol at $4^{\circ} \mathrm{C}$ after $24 \mathrm{~h}$ of exposure to siRNA-HLX2. RNase was used (Thermo Fisher Scientific, Inc.). Then, add the cell to -20 anhydrous ethanol, stir it at high speed while adding, discard the ethanol, add PBS at room temperature, place it for $15 \mathrm{~min}$, added $1 \mathrm{ml}$ Cell cycle staining kit (www.liankebio.com) and shake it for 5-10 sec, and incubate it at room temperature in dark for $30 \mathrm{~min}$, and the cell cycle was analyzed by flow cytometry (Flowjo ${ }^{\circledR}$ Flow data analysis software version 10.5.2, BD Biosciences).

Changes in associated proteins and genes after $H L X$ gene knockdown. STAT5 protein expression in NB4 cells was analyzed by western blotting after transfection with siRNA-HLX2, and the expression levels of related genes, including PAK1, NRPl and BTG1, were assessed by RT-qPCR.
Statistical analysis. The data were analyzed using SPSS 22.0 software (SPSS, Inc.). A Kolmogorov-Smirnov test was used to analyze data normality. Data are presented as the mean $\pm \mathrm{SD}$ of $\geq 3$ independent experiments. A t-test was used to compare the means of two groups. ANOVA (parametric) and Kruskal-Wallis (non-parametric) were used to compare the means of multiple groups, and variations of statistical significance were further subjected to post hoc pairwise analysis by applying the Tukey's test and the Dunn's test, respectively. $\mathrm{P}<0.05$ was considered to indicate a statistically significant difference.

\section{Results}

HLX expression is highest in the NB4 AML cell line. RT-qPCR was used to detect HLX gene expression in KG1a, NB4, THP-1 and Jurkat cells. It was found that the HLX gene was differentially expressed in KG1a (AML/M0 subtype), NB4 (AML/M3 subtype), and THP-1 (AML/M5 subtype) cells. Furthermore, HLX expression was higher in NB4 and THP-1 cells compared with the control group, but was highest in the 


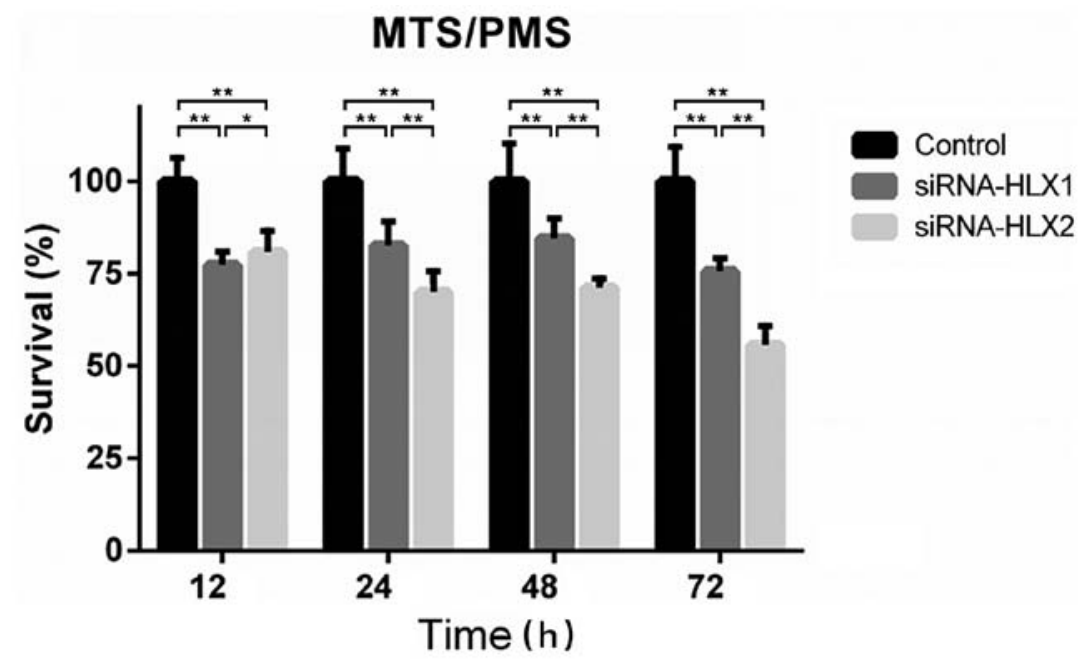

Figure 3. Comparison of the survival rates of NB4 cells at each time point after transfection of the high-GC siRNA, siRNA-HLX1 or siRNA-HLX2. ** $<0.01$. HLX, H2.0-like homeobox gene; siRNA, small interfering RNA.
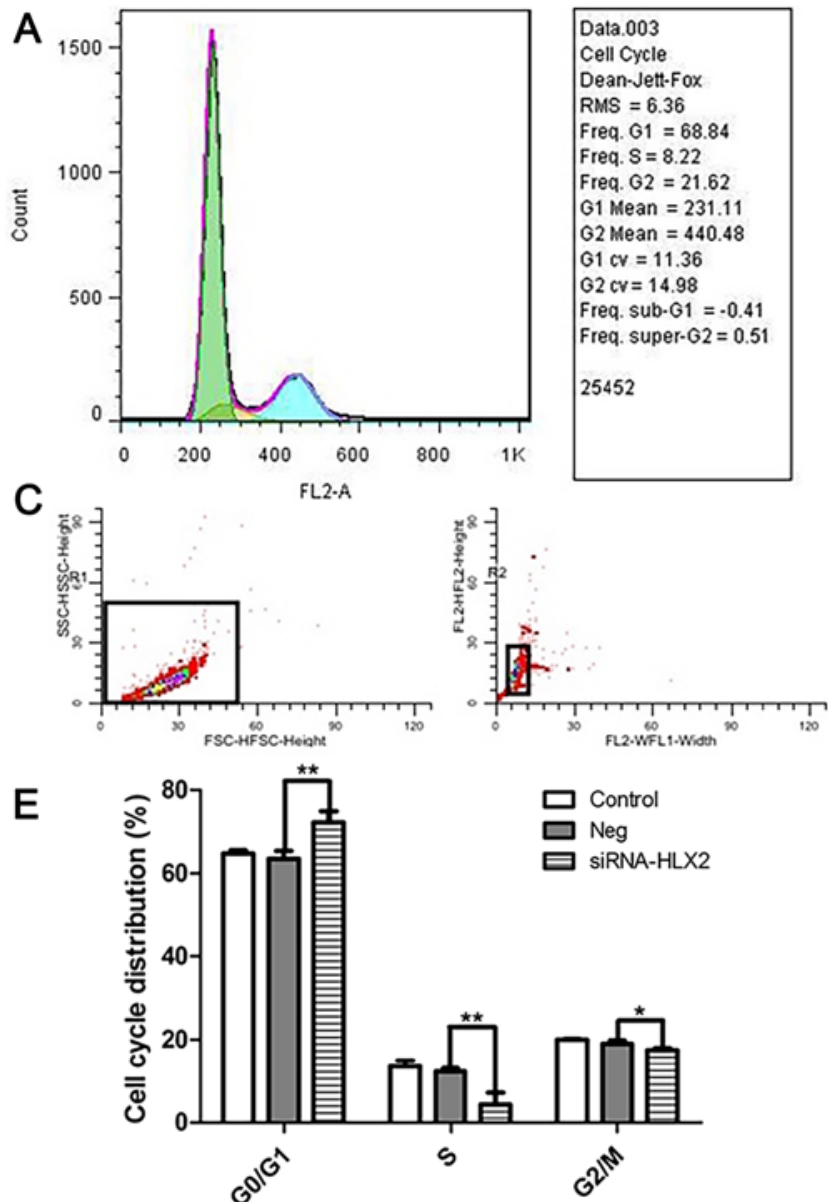
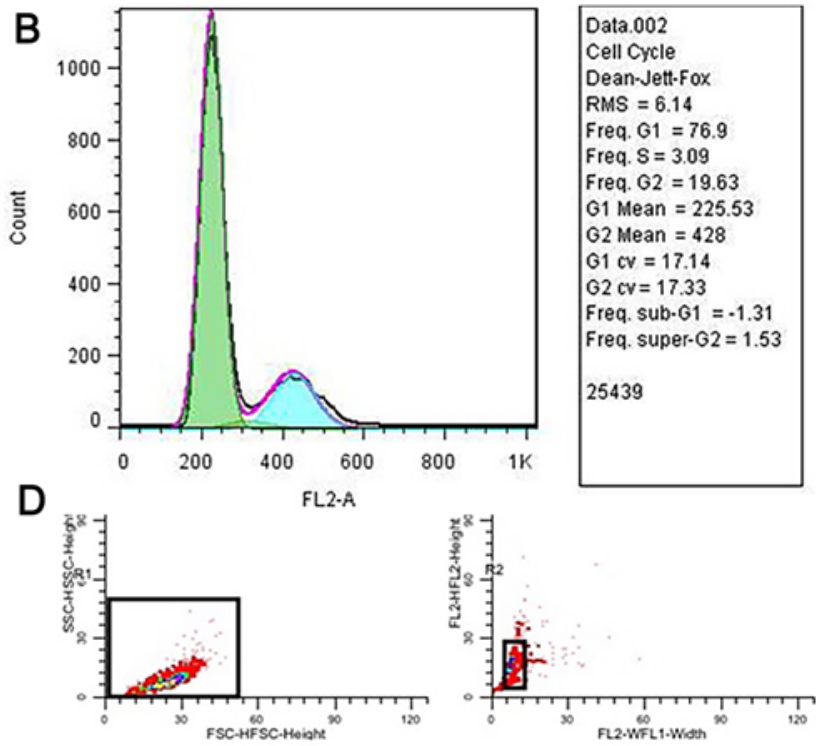

Figure 4. Flow cytometry analysis of the cell cycle distribution of NB4 cells transfected with siRNA-HLX2 for 24 h. (A) Negative control group. (B) Cells transfected with siRNA-HLX2 for $24 \mathrm{~h}$. (C) Scatter plot of the negative control group. (D) Scatter plot of the cells transfected with siRNA-HLX2 for $24 \mathrm{~h}$. (E) Cell cycle distribution. ${ }^{*} \mathrm{P}<0.05,{ }^{* *} \mathrm{P}<0.01$. HLX, H2.0-like homeobox gene; siRNA, small interfering RNA; Neg, negative control.

NB4 cell line (Fig. 1A). This result was also demonstrated by western blotting (Fig. 1B and C).

HLX expression is lowest at $24 \mathrm{~h}$ in NB4 cells after siRNA transfection. Cells in the blank control group (non-transfected), negative control group (neg siRNA) and experimental group (siRNA-HLX1 or siRNA-HLX2) were transfected as indicated, and HLX gene expression was detected using RT-qPCR after $12,24,48$ and $72 \mathrm{~h}$. HLX mRNA expression was lowest at $24 \mathrm{~h}$. Of the two gene-targeting sequences, siRNA-HLX2 was more 
A

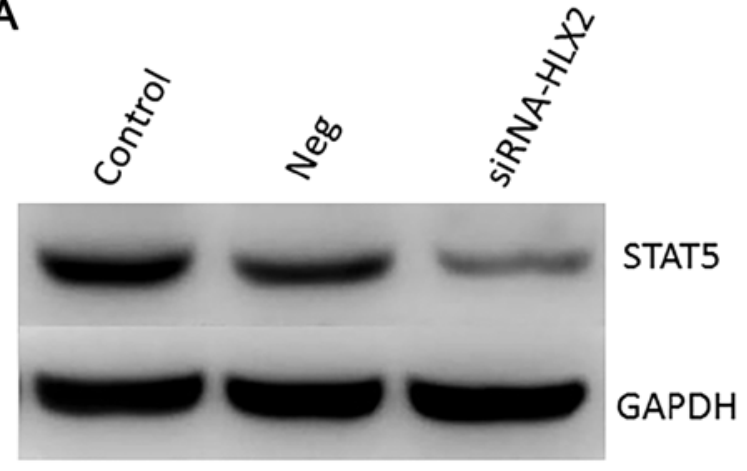

B

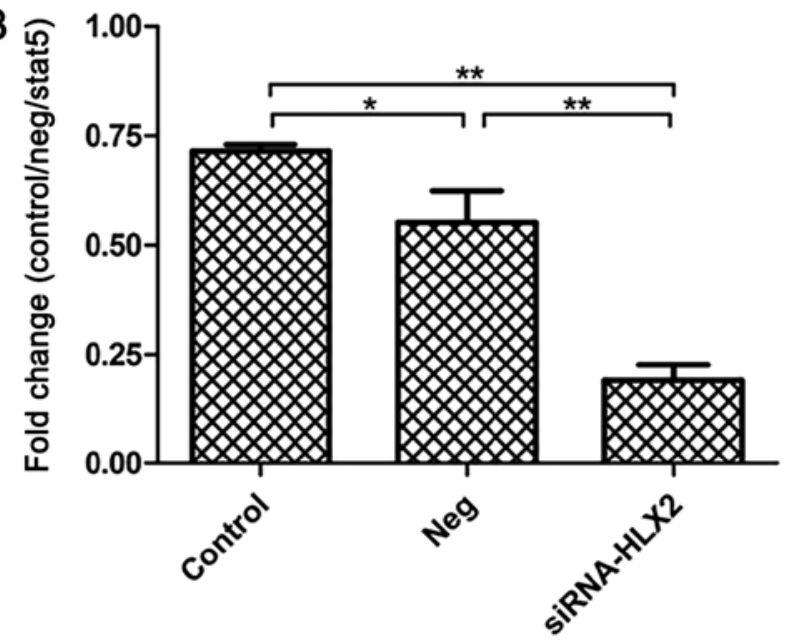

Figure 5. Changes in STAT5 protein expression in NB4 cells after siRNA-HLX2 transfection. (A) Comparison of STAT5 protein expression in NB4 cells after siRNA transfection. (B) Gray value analysis of western blotting results. ${ }^{*} \mathrm{P}<0.05,{ }^{* *} \mathrm{P}<0.01$. NS, not significant; HLX, H2.0-like homeobox gene; siRNA, small interfering RNA; Neg, negative control.

effective at silencing HLX expression than the siRNA-HLX1 group compared with the control group (Fig. 2A). HLX protein expression in the aforementioned groups was detected by western blotting after $24 \mathrm{~h}$, and the results indicated that HLX protein expression was decreased in the siRNA-HLX2 group compared with the untreated control group (Fig. 2B and C).

Knockdown of HLX using siRNA-HLX2 inhibits NB4 cell survival to a higher level compared with siRNA-HLXl. The absorbance at $490 \mathrm{~nm}$ was measured using the MTS/PMS method before transfection and at 12, 24, 48 and $72 \mathrm{~h}$ after transfection with siRNA-HLX1, siRNA-HLX2 and high-GC siRNA. The survival rate was calculated, and the results suggested that siRNA-HLX2 had a stronger inhibitory effect on proliferation compared with siRNA-HLX1. The survival rates of siRNA-HLX2 at $12,24,48$ and $72 \mathrm{~h}$ were $80.87 \pm 4.99$, $70.08 \pm 4.87,71.29 \pm 2.15$ and $55.73 \pm 4.46 \%$, respectively, compared with the control (Fig. 3).

Knockdown of HLX using siRNA-HLX2 arrests NB4 cells in the $G_{0} / G_{l}$ phase. As HLX gene expression was lowest at $24 \mathrm{~h}$ after siRNA-HLX2 transfection, the cell cycle distribution of NB4 cells transfected with siRNA-HLX2 was analyzed at $24 \mathrm{~h}$. At this time point, the $S$ phase population was reduced and the cell cycle was blocked in the $\mathrm{G}_{0} / \mathrm{G}_{1}$ phase. After transfection of NB4 cells with siRNA-HLX2, cell survival decreased with the reduction in the $\mathrm{S}$ phase population from $12.32 \pm 0.76$ to $4.29 \pm 2.85 \%, \mathrm{G}_{2} / \mathrm{M}$ phase population from $23.92 \pm 1.22$ to $20.13 \pm 2.56 \%$, and the number of cells in the $\mathrm{G}_{0} / \mathrm{G}_{1}$ phase increased from $63.57 \pm 1.87$ to $72.24 \pm 2.64 \%$ (Fig. 4).

STAT5 protein expression is downregulated in NB4 cells after siRNA-HLX2 transfection. After transfection of NB4 cells with siRNA-HLX2, STAT5 protein expression was detected by western blotting. In total, three groups were analyzed untreated control group, negative control group and siRNA-HLX2 group), and the results indicated that STAT5 protein expression decreased significantly in the siRNA-HLX2 group (Fig. 5). Compared with the untreated control and the non-targeting control, the STAT5 expression levels in the siRNA-HLX2 group decreased significantly.

Genes related to the JAK/STAT signaling pathway show altered expression in NB4 cells after siRNA transfection on the differences seen with time. The expression levels of JAK/STAT signaling pathway related genes (PAK1, NRP1 and BTG1) were detected by RT-qPCR after transfection of NB4 cells with siRNA-HLX1 or siRNA-HLX2. It was demonstrated that knockdown of the HLX gene decreased the expression levels of PAK1 (Fig. 6A and B) and NRP1 (Fig. 6C and D), but increased BTG1 expression (Fig. 6E and F).

\section{Discussion}

HLX is located on chromosome 1q41-q42.1, and its 1467-bp open reading frame encodes a protein composed of 488 amino acids (16). Previous studies have reported that the HLX gene participates in a variety of processes, including cell proliferation, differentiation and maturation $(17,18)$. Moreover, in combination with T-box transcription factor 21 and other transcription factors, HLX induces interferon- $\gamma$ production and provides T helper Th-2 cells with typical Th1-cell functions $(19,20)$. At present, previous studies have reported that the abnormal expression of HLX is closely related to the development of autoimmune diseases, such as Graves' disease, gastric cancer, colon cancer and other solid tumors (21), and that HLX plays an important role in the occurrence and development of leukemia (10). HLX is expressed in several types of leukemia but at different levels; for example, the expression of HLX is high in myeloid leukemia but low in lymphoid leukemia $(11,17)$.

AML is a malignant tumor, and individuals $<35$ years of age have the highest morbidity and mortality (22). The pathological mechanism of AML includes the abnormal clonal proliferation of leukemic cells, which affects normal hematopoiesis and endangers some organs and systems (10). Gene-targeting therapy has been revealed to improve the cure rate of AML (22); thus, it is critical to identify new gene 

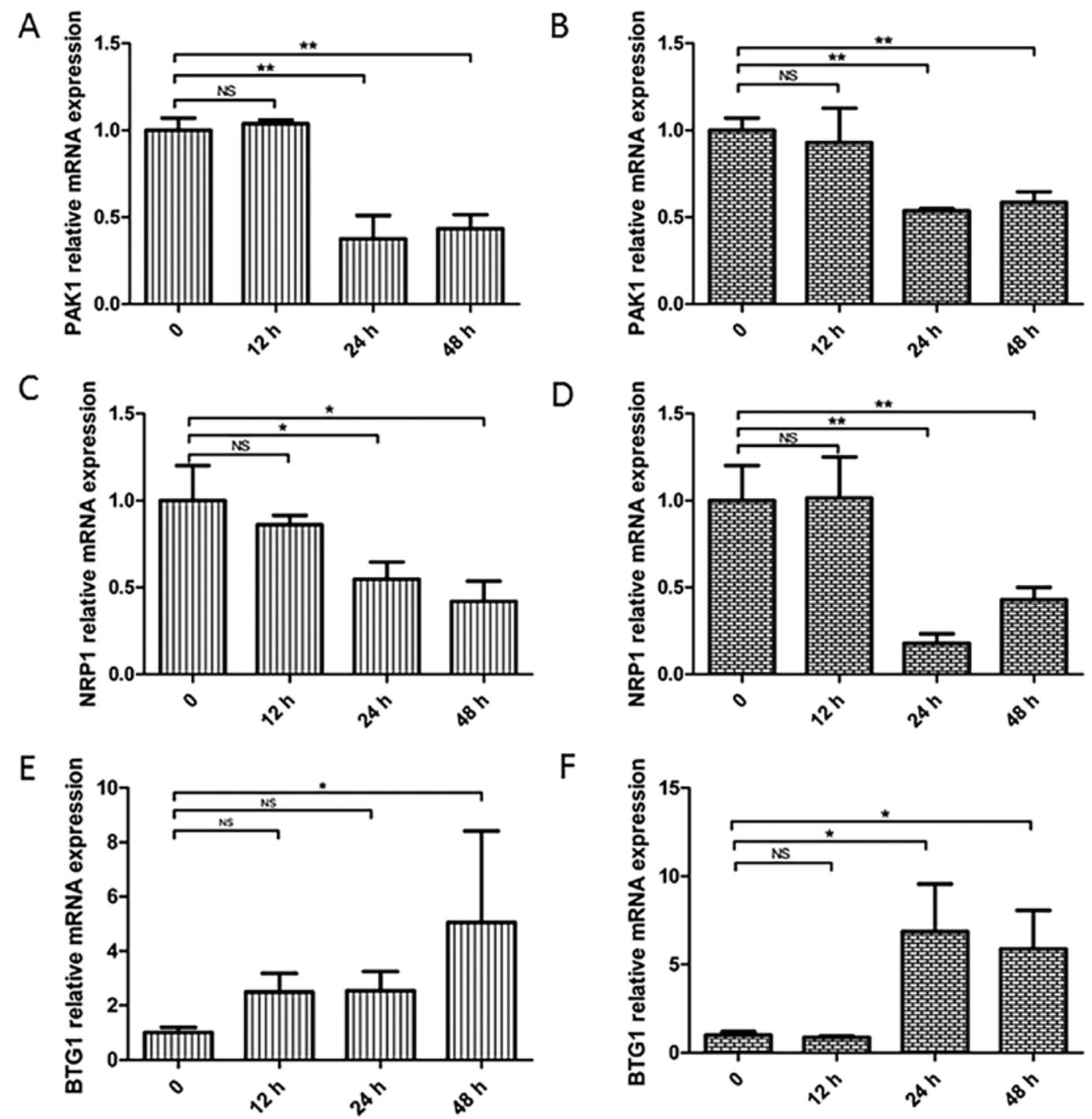

Figure 6. Changes in the related genes, PAK1, NRP1 and BTG1, after transfection of NB4 cells with siRNA-HLX1 or siRNA-HLX2. Data at $0 \mathrm{~h}$ were the scramble siRNA and are used as the control group. Changes in PAK1 gene expression in NB4 cells after (A) siRNA-HLX1 or (B) siRNA-HLX2 transfection. Changes in NRP1 gene expression in NB4 cells after (C) siRNA-HLX1 or (D) siRNA-HLX2 transfection. Changes in BTG1 gene expression in NB4 cells after (E) siRNA-HLX1 or (F) siRNA-HLX2 transfection. "P $<0.05,{ }^{* *} \mathrm{P}<0.01$. NS, not significant; HLX, H2.0-like homeobox gene; siRNA, small interfering RNA; Neg, negative control; PAK1, p21-activated kinase 1; BTG1, B-cell translocation gene 1; NRP1, neuropilin 1.

targets for AML. By analyzing HLX gene expression data from 354 patients with AML in the USA, Kawahara et al (11) found that the HLX gene was overexpressed in $87 \%$ of these patients and was associated with poor prognosis. Thus, these findings demonstrated that HLX may be a potential target in AML; however, to the best of our knowledge, few studies have reported the specific mechanism of HLX in AML.

The aim of the present study was to investigate the biological functions of HLX in AML cells. First, HLX expression was analyzed in AML cell lines of different subtypes and HLX was found to be differentially expressed in KG1a (AML/M0 subtype), NB4 (AML/M3 subtype) and THP-1 cells (AML/M5 subtypes), with the highest expression in the NB4 cell line. Then, after knocking down the HLX gene in NB4 cells using siRNA technology, the survival was assessed at 12, 24, 48 and $72 \mathrm{~h}$; cell proliferation was inhibited by HLX knockdown. The cell cycle was analyzed by flow cytometry, which identified an increased number of cells in $\mathrm{G}_{0} / \mathrm{G}_{1}$ phase and a decreased number in $\mathrm{S}$ phase, suggesting that the cell cycle was arrested at $G_{0} / G_{1}$ phase. Collectively, the present results indicated that the downregulation of HLX could block the cell cycle in $G_{0} / G_{1}$ phase, thus inhibiting the proliferation of AML cells.

The current study further investigated the signaling pathway affected by HLX that was involved in AML cell cycle regulation and proliferation. It was demonstrated that the knockdown of HLX resulted in a decreased expression of STAT5 at the protein level and of PAK1 and NRPl at the mRNA level, while BTG1 gene expression was increased. NRP1 is a receptor of VEGF $_{165}$ and can promote vascular proliferation via the PI3K/Akt, JAK/STAT and Notch signaling pathways (23-25). STAT5 is an important regulatory protein of the JAK/STAT signaling pathway and is closely associated with hematological malignancies (26). PAK1 is the downstream effector of HLX, regulates the carcinogenic effects of STAT5 in hematological disease $(27,28)$, and is involved in the pathogenesis of AML via the regulation of the MYC core network (12). Moreover, BTG1 is involved in a translocation with c-Myc and functions 
as a tumor suppressor gene in the BTG anti-proliferative protein family, leading to growth arrest or apoptosis in tumor cells $(13,14)$.

The JAK/STAT signaling pathway plays an important role in the development and progression of AML. Epidermal growth factor receptor (EGFR), a co-receptor of NRP1, is a receptor tyrosine kinase located upstream of this signaling pathway (24). Within this pathway, STAT5 is an important regulatory protein, and PAK1 and c-Myc are notable downstream target genes; furthermore, BTG1 is involved in the translocation of c-Myc (27). The findings of the present suggest that the HLX gene can regulate the JAK/STAT signaling pathway in AML, and after silencing HLX, genes associated with the JAK/STAT signaling pathway show altered expression over time. NRP1 expression was decreased, STAT5 protein expression was downregulated and the JAK/STAT signaling pathway was blocked, resulting in a reduction in PAK1 expression. Furthermore, under physiological conditions, STAT is known to regulate the reticular system via positive or negative feedback mechanisms involving the STAT5/c-Myc axis, and artificial mutagenesis of the STAT-binding site on c-Myc inhibits the JAK/STAT signaling pathway (29). Therefore, the increased expression of BTG1 may also lead to the negative feedback regulation of the JAK/STAT signaling pathway via the STAT5/c-Myc network, thus leading to the synergistic inhibition of STAT5 phosphorylation, repression of cell proliferation and cell cycle arrest.

Although the results are promising, the current study has several limitations. Since the HLX gene is closely related to genes such as BTG1, Forkhead Box O4, FYN proto-oncogene, Src family tyrosine kinase, growth arrest and DNA damage inducible $\alpha$, ras homolog family member B, Tumor Protein P63, ZFP36 Ring Finger Protein Like 1, Histone Deacetylase 7 and PAK1 (11), HLX may function similar to other homeobox proteins in AML to regulate several linked signaling pathways by affecting relevant upstream and downstream genes (30). The present study only examined the JAK/STAT pathway, and not investigate pathways that could be regulated by HLX. Additionally, the effects of HLX on cell differentiation and apoptosis were not fully elucidated. Thus, future studies will assess these processes in relation to HLX. The effects in the NB4 cell line was selected and the aim of the current study was to examine the relationship between the HLX gene and JAK signaling pathway. However, if the expression of HLX in cell lines is not high, the changes in related genes may not be obvious when HLX is downregulated. Thus, only the most representative cell line (NB4) was selected to be presented. In a subsequent study, HLX expression will be evaluated in AML primary cells, especially in the M3 subtype. To confirm the JAK/STAT signaling as the underlying mechanism of HLX, inhibitors of the JAK/STAT pathway will be used in future studies after downregulating HLX in AML cell lines, and the changes of JAK2 protein in NB4 cells will be measured after HLX knockdown. In addition, further studies will measure the phosphorylated STAT5 protein; the protein levels of genes, such as PAK1, NRP1 and BTG1, will also be evaluated.

In conclusion, the present results indicated that the HLX gene may be an important therapeutic target in AML and that it may play a critical role by regulating the JAK/STAT signaling pathway to regulate cell proliferation and cell cycle progression. Furthermore, the current study provides novel evidence of the pathogenic mechanism of HLX in AML and thus may help improve the treatment of AML (22).

\section{Acknowledgements}

The authors would like to thank Mr. Li-long Fan, Ms. Li-Hua Chen and Ms. Hai-Yan Lv (all Department of Central Laboratory, Taizhou Hospital of Zhejiang) for their instructive advice and useful suggestions on the manuscript. The authors would also like to thank Mr. Wei-Bo Zhao (Department of Orthopaedics, Taizhou Hospital of Zhejiang), Ms. Shuai-Shuai Chen and Ms. Jia-Xi Chen (both Department of Clinical Laboratory, Taizhou Hospital of Zhejiang) for their advice concerning the English language.

\section{Funding}

This work was supported by the Zhejiang Scientific Project of Health and Medicine, China (grant nos. 2014KYB309, 2017KY709 and 2017KY166), and the Zhejiang Province Foundation, China (grant no. 2016C33233).

\section{Availability of data and materials}

The datasets used and/or analyzed in the current study are available from the corresponding author upon reasonable request.

\section{Authors' contributions}

WDL designed most of the experiments. XYZ, QYG and LZ carried out the cell experiments. BGC and MZ helped design the experiments. LYW, DQZ and YPS analyzed the data. XYZ wrote the manuscript. All authors read and approved the final manuscript.

\section{Ethics approval and consent to participate}

Not applicable.

\section{Patient consent for publication}

Not applicable.

\section{Competing interests}

The authors declare that they have no competing interests.

\section{References}

1. Seifert A, Werheid DF, Knapp SM and Tobiasch E: Role of Hox genes in stem cell differentiation. World J Stem Cells 7: 583-595, 2015.

2. Casaca VI, Illi S, Suttner K, Schleich I, Ballenberger N, Klucker E, Turan E, Mutius EV, Kabesch M and Schaub B: TBX21 and HLX1 polymorphisms influence cytokine secretion at birth. PLoS One 7: e31069, 2012.

3. Rajaraman G, Murthi P, Pathirage N, Brennecke SP and Kalionis B: Downstream targets of homeobox gene HLX show altered expression in human idiopathic fetal growth restriction. Am J Pathol 176: 278-287, 2010.

4. Prahst C, Kasaai B, Moraes F, Jahnsen ED, Larrivee B, Villegas D, Pardanaud L, Pibouin-Fragner L, Zhang F, Zaun HC, et al: The H2.0-like homeobox transcription factor modulates yolk sac vascular remodeling in mouse embryos. Arterioscler Thromb Vasc Biol 34: 1468-1476, 2014. 
5. Lu ZY, Zhong NS, Xie Y and Hu PJ: Internal Medicine. People's Health Press, China, pp 563-674, 2011. (In Chinese).

6. Leukemia \& Lymphoma Group, Chinese Society of Hematology, Chinese Medical Association: The Guidelines for Diagnosis and Treatment of Acute Myelogenous Leukemia (Relapse/ Refractory) in China (2017). Zhonghua Xue Ye Xue Za Zhi 38: 183-184, 2017 (In Chinese).

7. Luo B, Que ZJ, Zhou ZY, Wang Q, Dong CS, Jiang Y, Hu B, Shi H, Jin Y, Liu JW, et al: Feiji recipe inhibits the growth of lung cancer by modulating T-cell immunity through indoleamine-2,3-dioxygenase pathway in an orthotopic implantation model. J Integr Med 19: 283-289, 2018.

8. Ghasemi R, Struthers H, Wilson ER and Spencer DH: Contribution of CTCF binding to transcriptional activity at the HOXA locus in NPM1-mutant AML cells. Leukemia: May 12, 2020 (Epub ahead of print).

9. Zhang Y, Zhang YM, Zhang YS, Tang GS, Zhang WP, Yang JM, Wang JM and $\mathrm{Hu}$ XX: Prognostic significance of minimal residual disease before post-remission therapy in younger adult acute myeloid leukemia patients with intermediate risk and negative of FLT3-ITD, NPM1 and biallelic CEBPA mutations. Zhonghua Xue Ye Xue Za Zhi 40: 597-601, 2019 (In Chinese).

10. Pandolfi A and Steidl U: HLX in AML: Novel prognostic and therapeutic target. Oncotarget 3: 1059-1060, 2012.

11. Kawahara M, Pandolfi A, Bartholdy B, Barreyro L, Will B, Roth M, Okoye-okafor UC, Todorova TI, Figueroa ME, Melnick A, et al: H2.0-like homeobox (HLX) regulates early hematopoiesis and promotes acute myeloid leukemia. Cancer Cell 22: 194-208, 2012.

12. Pandolfi A, Stanley RF, Yu Y, Bartholdy B, Pendurti G, Gritsman K, Boultwood J, Chernoff J, Verma A and Steidl U: PAK1 is a therapeutic target in acute myeloid leukemia and myelodysplastic syndrome. Blood 126: 1118-1127, 2015.

13. Van Galen JC, Kuiper RP, Van EL, Levers M, Tijchon E, Scheijen B, Waanders E, van Reijmersdal SV, Gilissen C, van Kessel AG, et al: BTG1 regulates glucocorticoid receptor autoinduction in acute lymphoblastic leukemia. Blood 115: 4810-4819, 2010

14. Zheng HC, Li J, Shen DF, Yang XF, Zhao S, Wu YZ, Takano Y, Sun HZ, Su RJ, Luo JS and Gou WF: BTG1 expression correlates with pathogenesis, aggressive behaviors and prognosis of gastric cancer: A potential target for gene therapy. Oncotarget 6 : 19685-19705, 2015.

15. Livak KJ and Schmittgen TD: Analysis of relative gene expression data using real-time quantitative PCR and the 2(-Delta Delta C(T)) method. Methods 25: 402-408, 2001.

16. Fröhling S: Widespread over-expression of the non-clustered homeobox gene HLX in acute myeloid leukemia. Haematologica 97: 1453, 2012

17. Testori J, Schweighofer B, Helfrich I, Sturtzel C, Lipnik K, Gesierich S, Nasarre P, Hofer-Warbinek R, Bilban M, Augustin HG and Hofer E: The VEGF-regulated transcription factor HLX controls the expression of guidance cues and negatively regulates sprouting of endothelial cells. Blood 117: 2735-2744, 2011

18. Yamakawa T, Sato Y, Matsumura Y, Kobayashi Y, Kawamura Y, Goshima N, Yamanaka S and Okita K: Screening of human cDNA library reveals two differentiation-related genes, HHEX and HLX, as promoters of early phase reprogramming toward pluripotency. Stem Cells 34: 2661-2669, 2016.
19. Zheng WP, Zhao Q, Zhao X, Li B, Hubank M, Schatz DG and Flavell RA: Up-regulation of Hlx in immature Th cells induces IFN-gamma expression. J Immunol 172: 114-122, 2004.

20. Xu Y, Gao J, Su Z, Dai X, Li Y, Liu Y, Chen J, Tong J, Zhang Y, Wu C, et al: Downregulation of Hlx closely related to the decreased expressions of T-bet and Runx 3 in patients with gastric cancer may be associated with a pathological event leading to the imbalance of Th1/Th2. Clin Dev Immunol 2012: 949821, 2012.

21. Morita $M$, Watanabe $M$, Inoue $N$, Inaoka $C$, Akamizu $T$, Tatsumi KI, Hidaka Y and Iwatani Y: Functional polymorphisms in TBX21 and HLX are associated with development and prognosis of Graves' disease. Autoimmunity 45: 129-136, 2012.

22. Prada-Arismendy J, Arroyave JC and Röthlisberger S: Molecular biomarkers in acute myeloid leukemia. Blood Rev 31: 63-76, 2017.

23. Li L, Jiang X, Zhang Q, Dong X, Gao Y, He Y, Qiao H, Xie F, $X i e X$ and Sun $X$ : Neuropilin-1 is associated with clinicopathology of gastric cancer and contributes to cell proliferation and migration as multifunctional co-receptors. J Exp Clin Cancer Res 35: 16, 2016

24. Hendricks C, Dubail J, Brohée L, Delforge Y, Colige A and Deroanne C: A novel physiological glycosaminoglycan-deficient splice variant of neuropilin-1 is anti-tumorigenic in vitro and in vivo. PLoS One 11: e0165153, 2016.

25. Piechnik A, Dmoszynska A, Omiotek M, Mlak R, Kowal M, Stilgenbauer S, Bullinger L and Giannopoulos K: The VEGF receptor, neuropilin-1, represents a promising novel target for chronic lymphocytic leukemia patients. Int J Cancer 133: 1489-1496, 2013.

26. Wang Z and Bunting KD: STAT5 activation in B-cell acute lymphoblastic leukemia: Damned if you do, damned if you don't. Cancer Cell Microenviron 3: e1186, 2016.

27. Eswaran J, Li DQ, Shah A and Kumar R: Molecular pathways: Targeting P21-activated kinase 1 signaling in cancer-opportunities, challenges and limitations. Clin Cancer Res 18: 3743-3749, 2012.

28. Chatterjee A, Ghosh J, Ramdas B, Mali RS, Martin H, Kobayashi M, Vemula S, Canela VH, Waskow ER, Visconte V, et al: Regulation of Stat 5 by FAK and PAK 1 in oncogenic FLT3- and KIT-driven leukemogenesis. Cell Rep 9: 1333-1348, 2014.

29. Yamada O and Kawauchi K: The role of the JAK-STAT pathway and related signal cascades in telomerase activation during the development of hematologic malignancies. JAKSTAT 2: e25256, 2013.

30. Breitinger C, Maethner E, Garciacuellar MP and Slany RK: The homeodomain region controls the phenotype of HOX-induced murine leukemia. Blood 120: 4018-4027, 2012.

This work is licensed under a Creative Commons Attribution-NonCommercial-NoDerivatives 4.0 International (CC BY-NC-ND 4.0) License. 